\title{
The study of algorithms for 3D's engineering object representing with regard to their construction specifics
}

\author{
Ekaterina Sokolova ${ }^{1, *}$ \\ ${ }^{1}$ North Caucasian Institute of Minning and Metallurgy, 362021, Vladikavkaz, RNO-Alania, Russia
}

\begin{abstract}
This work deals with algorithms of 3D object representation with due regard to their construction specifics. The criterion of the integral adjacency of an unambiguous/fuzzy truth value to the absolute truth value is analyzed.
\end{abstract}

\section{Formulation of the problem}

The development of information technology it is especially effective to use threedimensional models in the industry, mechanical engineering. To optimize the representation of a three-dimensional object, it is necessary to take into account the features of its design.

\section{Multicriteria convex programming problems}

The system (4.3) is a formal representation of the multicriterion problem [4-7], which cannot be expressed with a single indicator.

Let us consider the system developed on the basis of the algorithm, as a system with several target parameters. When implementing the algorithm for saving a 3D image, the volume of the resulting file tends to the minimum, the quality of the resulting image tends to the maximum, the selected subset of fragments tends to the minimum. In the case of a deeper analysis, additional criteria may appear [1]. As can be seen from the conditions of the task, one of the criteria must be turned to the maximum value, and the other criterion must be turned to the minimum.

Let us try to present the desired indicators in the form of some controlled parameters, for example, $a_{1}, a_{2}, a_{3}, \ldots, a_{r}$; then the whole task will be to find point $\vec{a}^{(0)} \in A$. The specified point will allow receiving a universal result for all indicators at once if they are presented in the form of function $F_{1}(\vec{x}), F_{2}(\vec{x}), \ldots, F_{r}(\vec{x})$. Obviously, this problem has no solution for the general case. The only solution is to choose the optimal combination of indicators that fit each criterion. Search for these combinations is carried out by trial and error or intuitively. A certain value $R$ can be so large that its growth does not affect the extremum point (min). At present, it is possible to easily calculate the values of $R$ by

* Corresponding author: katya sea@mail.ru 
means of convex programming problems [2]. To reduce the complexity that can be caused by a gradient break, it is recommended to use the following:

$$
S_{R}\left(x_{1}, x_{2}, \ldots, x_{n}\right)=f\left(x_{1}, x_{2}, \ldots, x_{n}\right)+R \sum_{j=1}^{m} \mu^{2}\left(\varphi_{j}\left(x_{1}, x_{2}, \ldots, x_{n}\right)\right),
$$

applying at that the quadratic penalty function. Depending on the conditions of the problem, the function $S_{R}$ can be smooth, then it is quite easy to find its minimum with the help of a set of gradient methods. However, for large values of $R$, the calculation is slow, and the obtained results are not accurate.

Next, let us turn to the consideration of multicriterion problems, which is directly applicable to the problem stated in this paper.

With a single target parameter, expressed through parameters that can be controlled, only one objective function is obtained.

In computer graphics, similar problems [7] arise during processing small images and with minimum requirements to the result. In the case of processing $3 \mathrm{D}$ images obtained by scanning in real time mode and requiring immediate processing, the interaction of many parameters is detected, and it is obvious that the efficiency of their work cannot be expressed in one parameter.

In our case it is desirable to get an image of the highest quality with a minimum size, minimal artefacts within minimal time.

When processing each particular object, it is likely that additional parameters will be detected. As follows from the above, many indicators, some of which tending to a maximum, others tending to a minimum, are a typical example of a multicriterion problem [3].

\section{Pareto solutions}

Let us denote all parameters of interest as $\left(x_{1}, x_{2}, \ldots, x_{n}\right)$.

Our task will be to search for a global extremum [5] for a certain point for several functions $f(x), f_{2}(x), \ldots, f_{k}(x)$ at once.

It is obvious that the stated problem has no solution for the general case. In order to choose the optimal solution [7], it is necessary to find such variants that will be suitable for all criteria at once.

To make this choice, you need to apply additional parameters. As is known, in set $M$ one can select a subset $p$ of the so-called Pareto solutions, from which the choice of the compromise variant is made.

Further, let us introduce the definition [6] that for each multicriterion problem there is a Pareto solution: this is a certain point which allows achieving the desired best value for one of the parameters, while the other parameters do not change for the worse.

Therefore, the given point is characterized by such a property that if $f i(x)>f i(x)$ holds, then one can find such $j(1 \leq j \leq k)$ that $f j(x)<f j(x)$.

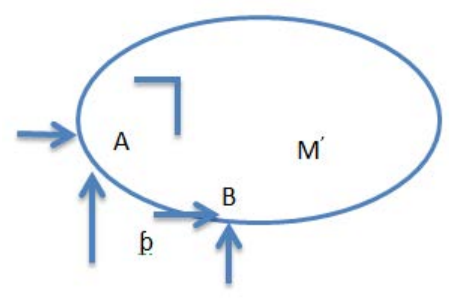

Fig. 1. Example of Pareto solutions. 
Based on the definition, we can make the following conclusion: Pareto solution is a solution that surpasses all possible solutions to meet the requirements of the conditions and limitations of the system. In most cases, the resulting set of Pareto solutions [4] is less than the given set.

Let's consider an example: let there be two criteria in the given condition, therefore, for each value of $x$ that enters the set, there is a corresponding point with the coordinates obtained from these functions. As seen from the figure, the Pareto solutions are located along arc $A B$. However calculating Pareto solutions is a challenging task. Below we consider the possible solutions, which are applicable to the conditions of the research problem.

$$
\sum_{i=1}^{k} a_{i} f_{i}(\bar{x}) \rightarrow \min , \bar{x} \in M,
$$

where $a_{i}$ is a weight number (positive number). This element determines the importance of the criteria. There is direct relation between them: the higher the coefficient, the more is the importance, and vice versa: the lower the ratio, the less is the importance. It should be noted that in the case of all convex functions, the minimum point always belongsto the Pareto set of solutions.

Similarly, from the set of Pareto solutions it is easy to obtain the minimum point. The above-described coefficients are selected experimentally.

The next way is to choose the main indicator [1,6]. Heuristics is applied again. And the main indicator is chosen based on experiments or expert opinion. The chosen main indicator must be brought to either a minimum or a maximum depending on the conditions of the task. The remaining indicators change, but within the specified range, thus the set goal is achieved.

It is also possible to use the method of successive concessions, when all available functions are arranged in descending order, depending on their significance for a particular task. The solution is searched from the end of the sorted list. Further it is necessary to rise higher and each time to change the values according to the conditions of the current criterion, thus at each step a new criterion is obtained. The resulting point will be considered to be the solution of the problem.

\section{Conclusion}

In the resuscitation of research in the work, the algorithms for the $3 \mathrm{D}$ engineering object were studied, taking into account their structural features. Also, the effectiveness of multicriterial tasks, issued for solving the following problems, was confirmed: solutions for saving and processing.

From the above it is obvious that for each multicriterion task, it is necessary for the user to participate in assessing the importance of the criteria.

\section{References}

1. Zhi-liang Zhu, Wei Zhang, Kwok-wo Wong and Hai Yu Inf. Sci. 6 1171-86 (2011)

2. Wei Zhang, Kwok-wo Wong, Hai Yu and Zhi-liang Zhu Commun. Nonlinear Sci. Numer. Simul. 3 584-600 (2013)

3. Xingyuan Wang, Lin Teng and Xue Qin 2012 Signal Process 4 1101-08 (2012)

4. Yicong Zhou, Weijia Cao, and Philip Chen Signal Process 8 197-207 (2014)

5. Sokolov A A, Miroshnikov A S, Sokolova E A Gornyi Zhurnal 12 83-86 (2016) 
6. Murillo-Escobar M A, Cruz-Hernández C, Abundiz-Pérez F López-Gutiérrez R M and Acosta Del Campo O R Signal Process 109 119-131 (2015)

7. Kumaritov A M., Sokolova E A, Sokolov A A Gornyi Zhurnal 2 94-96 (2016)

8. Yingqian Zhang and Xingyuan Wang Inf. Sci. 20 329-351 (2014)

9. Wei Zhang, Kwok-wo Wong, Hai Yu and Zhi-liang Zhu Commun. Nonlinear Sci. Numer. Simul. 8 2066-80 (2013)

10. V. Zhukov, E. Masyutkin, B. Avdeyev, The application of mathematical modeling for the development of devices as an example of viscous fluid purification from magnetic impurity, IOP Conference Series: Materials Science and Engineering, № 177, 012015. (2017) DOI: 10.1088/1757-899X/177/1/012015

11. B. Avdeyev, E. Masyutkin, S. Golikov, S. Sokolov and V. Gavrilov, Calculation of efficiency curve of magnetic hydrocyclone, 2017 IEEE Conference of Russian Young Researchers in Electrical and Electronic Engineering (EIConRus), St. Petersburg, Pp. 1225-1228 (2017) DOI:10.1109/EIConRus.2017.7910783.

12. B. Avdeyev, Development of criteria for a magnetic hydrocyclones, MATEC Web Conferences Vol. 129, 06012 (2017) DOI:https://doi.org/10.1051/matecconf/2017129060121.

13. Sokolova, E.A., Aslanov, G.A., Sokolov, A.A., A modern approach to storing of $3 D$ geometry of objects in machine engineering industry, IOP Conference Series: Materials Science and Engineering, 177, 012036 (2017)

14. Sokolova, E., The study of the possibility of using innovative technologies for saving engineering 3d objects, MATEC Web of Conferences , 129, 03008 (2017)

15. Sokolova, E.A., Dzhioev, G.A, Development of an algorithm for automated enhancement of digital prototypes in machine engineering, IOP Conference Series: aterials Science and Engineering, 177, 012037 (2017)

16. Zhi-liang Zhu, Wei Zhang, Kwok-wo Wong and Hai Yu ,Nonlinear Sci. Numer. Simul, Inf. Sci., 3, 16 (2013) 